\title{
Factores demográficos asociados al tratamiento oportuno en mujeres con cáncer de mama, Antioquia
}

\section{Demographic factors associated with timely treatment in women with breast cancer, Antioquia}

Carlos Gomez-Mercado (iD, Ángela Segura-Cardona² (D), Dubán Pájaro-Cantillo3 ${ }^{(D)}$, Miler Mesa-Largo 4 (D), Martha Cadavid$\mathrm{Gil}^{5}$ (D)

1. Universidad CES. Medellín, Colombia. Correo: klargomez@gmail.com - https://orcid.org/0000-0003-4123-2812

2. Universidad CES. Medellín, Colombia. Correo: asegura@ces.edu.co - https://orcid.org/0000-0002-0010-1413

3. Universidad CES. Medellín, Colombia. Correo: dpajaro@ces.edu.co - https://orcid.org/0000-0002-3728-8699

4. Universidad CES. Medellín, Colombia. Correo: mmeza@ces.edu.co - https://orcid.org/0000-0001-5177-6325

5. Secretaría Seccional de Salud y Protección Social de Antioquia. Medellín, Colombia. Correo: marta.cadavid@antioquia.gov.co - https://orcid.org/00000002-0790-7444

Tipología: Artículo de investigación científica y tecnológica

Para citar este artículo: Gomez-Mercado C, Segura-Cardona A, Pájaro-Cantillo D, Mesa-Largo M, Cadavid-Gil M. Factores demográficos asociados al tratamiento oportuno en mujeres con cáncer de mama, Antioquia. Duazary. 2020 julio - septiembre; 17(3): 25 - 33. Doi: http://dx.doi.org/10.21676/2389783X.3318

\section{RESUMEN}

Palabras

clave:

neoplasias de

la mama;

mujeres;

Colombia.
El cáncer de mama es la principal causa de muerte en el mundo por enfermedades tumorales malignas entre las mujeres y el pronóstico de una mujer con esta enfermedad depende de un diagnóstico y tratamiento integral. Se estableció la asociación entre los factores demográficos y el tratamiento oportuno de las mujeres con cáncer de mama de Antioquia en el primer semestre 2017. Se realizó un estudio transversal, retrospectivo, para identificar la incidencia del cáncer de mama y la asociación de los determinantes demográficos no causales de los pacientes con cáncer de mama y la oportunidad en el tratamiento, mediante una regresión logística binaria múltiple. Se presentaron 355 casos nuevos de cáncer de mama, para una tasa de incidencia de 10,5 casos por cada 100.000 mujeres. Los factores demográficos asociados fueron: residencia urbana (RPa: 3,08 IC95\%: 0,38-24,83), afiliación al régimen subsidiado (RPa: 1,90 IC95\%: 0,81-4,46), episodios previos de hospitalización (RPa: 1,90 IC95\%: 0,814,46), diagnóstico tardío $\geq 16$ días (RPa: 0,46 IC95\%: 0,18-1,19) y la edad (RPa: 0,97 IC95\%: 0,95-1,00). EI tratamiento oportuno de pacientes de cáncer de mama se asocia con la residencia urbana, la afiliación al régimen subsidiado, episodios previos de hospitalización, un diagnóstico tardío y la edad, pero estas asociaciones no son estadísticamente significativas.

\section{ABSTRACT}

Keywords: Breast Cancer; Women; Colombia.
Breast cancer is the leading cause of death in the world due to tumor diseases among women and the prognosis of a woman with this disease. This is an association between demographic factors and the timely treatment of women with breast cancer in Antioquia in the first half of 2017. A cross-sectional, retrospective study is carried out to identify the incidence of breast cancer and the association of Demographic Determinants There are no causes of patients with breast cancer and the opportunity in the treatment. There are 355 cases of breast cancer, for an incidence rate of 10.5 cases per 100,000 women. The associated demographic factors were: urban residence (RPA: 3.08 IC95\%: 0.38-24.83), affiliation to the subsidized regime (RPA: 1.90 IC95\%: 0.81-4.46), previous episodes of hospitalization (RPa: 1.90 IC95\%: 0.81-4.46), late diagnosis $\geq 16$ days (RPa: 0.46 IC95\%: 0.18-1.19) and age (RPa: 0, 97 $95 \% \mathrm{Cl}: 0.95-1.00)$. Timely treatment of cancer patients is associated with urban residence, affiliation to the subsidized regimen, previous episodes of hospitalization, late diagnosis and age, but these associations are not statistically significant. 


\section{INTRODUCCIÓN}

El cáncer de mama es la principal causa de muerte en el mundo por enfermedades tumorales malignas entre las mujeres ${ }^{1}$, y se ha considerado como problema de los países desarrollados, con tasas de incidencia hasta las 113 casos por 100.000 mujeres al año; sin embargo, en las últimas décadas, en países en vía de desarrollo como Colombia, se observa una tendencia creciente de esta enfermedad, por lo cual, las autoridades sanitarias han decidido incluirla entre las prioridades de salud pública ${ }^{2,3}$.

Según las estadísticas del proyecto del Observatorio Mundial del Cáncer (GLOBOCAN, por sus siglas en inglés) de la Organización Mundial de la Salud (OMS), la tasa de incidencia estimada en el 2017 para Suramérica fue de 53 casos por 100.000 mujeres y en Colombia de 34 casos por cada 100.000 mujeres al año ${ }^{1}$. De acuerdo a lo reportado por Instituto Nacional de Cancerología (INC) de Colombia en su anuario estadístico 2015, en esta institución prestadora de servicios de salud se diagnosticaron 685 casos nuevos de cáncer de mama en mujeres ${ }^{4}$.

Desde la década de los noventa, en una revisión sistemática liderada por Richard ${ }^{5}$ y publicada en Lancet, demostró que la sobrevida de las mujeres con cáncer de mama estaba relacionada con el diagnóstico y tratamiento oportuno en un tiempo inferior a 90 días. De otra parte, se ha evidenciado que las mujeres con mayor vulnerabilidad económica o social enfrentan mayores dificultades para acceder oportunamente al servicio de salud, lo cual, termina incidiendo en el inicio del tratamiento y por ende, modifican el pronóstico de su enfermedad ${ }^{6,7}$.

En Colombia, las mujeres con cáncer de mama enfrentan un sistema burocrático que obliga a recorrer un itinerario de trámites administrativos, clínicos y legales, en busca de la atención oportuna ${ }^{8}$, por lo cual, muchos pacientes no logran obtener un diagnostico oportuno? ${ }^{9}$.

Por lo anterior, se propone un estudio con el objetivo de establecer la asociación entre los factores demográficos y el tratamiento oportuno de las mujeres con cáncer de mama del departamento de Antioquia en el 2017, que dado que es un tema de interés actual, por las implicaciones políticas, sociales, económicas y desde la salud pública y fortalecer la evidencia existente

\section{MATERIALES Y MÉTODOS}

\section{Tipo de estudio}

Estudio transversal, observacional, descriptivo, retrospectivo y analítico, para la identificación de la incidencia de cáncer de mama y la asociación entre determinantes demográficos no causales de los pacientes con cáncer de mama del departamento de Antioquia en el primer semestre del 2017 y la oportunidad en el tratamiento.

\section{Población y muestra}

Se analizaron los registros tomados del Sistema de Vigilancia en Salud Pública (SIVIGILA) 2017 para el evento de vigilancia en salud pública de cáncer de mama, previa autorización formal de la Secretaría Seccional de Salud y Protección Social de Antioquia (SSSYPSA). Se realizó censo de todos los registros, se diseñó un instrumento en Excel para la captura de las variables de interés y como medida para controlar los sesgos de información, se excluyeron aquellos que tenían datos faltantes por encima del 10\%. En total se incluyeron 355 registros para el estudio.

\section{Variables}

En el estudio, la variable dependiente fue la oportunidad en el tratamiento de las pacientes con cáncer de mama; entendida como la media de tiempo que transcurre desde la fecha de resultado de la prueba diagnóstica que sugiere al médico la probabilidad de cáncer y/o la fecha de resultado de prueba confirmatoria, hasta el inicio del tratamiento con atención médica con sus respectivas ayudas diagnósticas y terapéuticas, esta variable fue dicotomizada así: tratamiento oportuno ( $\leq 30$ días); como categoría de riesgo y tratamiento no oportuno ( $\geq 31$ días) como categoría de referencia. Las variables independientes incluidas fueron: área de residencia (urbana, rural), régimen de afiliación en salud (contributivo/especial, subsidiado), hospitalización 
previa (si, no), condición final (viva, muerta), edad en años y días de oportunidad diagnóstica.

\section{Análisis de datos}

En cuanto a las técnicas de procesamiento y análisis de los datos, se realizó en primera instancia un plan de edición de los datos, donde se codificaron las preguntas abiertas a través de la categorización de las respuestas descritas en los registros del SIVIGILA. Además, se identificaron las respuestas inconsistentes, los datos faltantes o respuestas como (no aplica, no sabe, no responde) con el fin de categorizarlas e incluirlas en el estudio. Posteriormente, se realizó la exploración de los datos, con el propósito de observar la forma de la distribución de las variables, buscar las relaciones entre los datos, crear variables adicionales en caso de ser necesario, recategorizar variables y observar los comportamientos de subgrupos importantes. La digitación de los datos se realizó en el software SPSS $^{\circledR}$ versión 21 (licencia Universidad CES).

Se ejecutó un análisis descriptivo de todas las variables, calculando de medidas de frecuencia y medidas de resumen. Para el análisis bivariado se utilizó la prueba Chi-Cuadrado de Independencia $\left(X^{2}\right)$ de Pearson, con el fin de establecer asociaciones bivariadas entre el tratamiento oportuno y las demás variables, dónde se hallaron razones de prevalencia crudas, intervalos de confianza y valores $p$. Además, se exploró si hubo diferencias significativas entre las medianas de días de oportunidad en el tratamiento según régimen de afiliación, con la prueba de $W$ de Mann Whitney. Por último, se realizó análisis multivariado por medio de regresión logística binaria múltiple utilizando el método introducir, donde se incluyeron variables con todas las variables y se calcularon razones de prevalencia ajustadas por las variables independientes, sus intervalos de confianza del $95 \%$ y el nivel de significancia estadística, el cual, se fijó con un valor $p<0,05$.

\section{Declaración sobre aspectos éticos}

Se siguieron las pautas de la Declaración del Helsinki de 1975 y según lo reglamentado en la Resolución 8430 de 1993 del Ministerio de Salud de Colombia, el estudio fue catalogado como sin riego, pues se trabajó con fuente de información secundaria. Los resultados de este estudio generan evidencia científica para la toma de decisiones en salud pública.

\section{RESULTADOS}

En total se analizaron 355 registros de mujeres con cáncer de mama del departamento de Antioquia. Se observó que el $95,2 \%$ de las pacientes vivían en zona urbana, el $78,2 \%$ pertenecían al régimen contributivo o especial en salud, el $97,2 \%$ de ellas fueron hospitalizadas previo al inicio del tratamiento y el 1,7\% fallecieron en el 2017.

Se aplicó prueba de normalidad de Kolmogorov Smirnov, para probar si las variables cuantitativas, provenían de una población con distribución normal y se comprobó que la edad $(p=0,085)$ tenía una distribución normal, contrario a la oportunidad en el diagnóstico $(p=0,00)$ y la oportunidad en el tratamiento $(p=0,000)$. Además, al categorizar la oportunidad en el tratamiento, se evidenció que el sólo el $22,8 \%$ recibió tratamiento oportuno. Se observó que el promedio de edad de las mujeres fue de 57 años con una desviación estándar de 18 años. Además, el $50 \%$ de la población de estudio tuvo una oportunidad diagnostica de 5 días o menos con un rango intercuartíl (RIQ) de 6 días, mientras que el $50 \%$ de las participantes tuvo oportunidad en el tratamiento de 72 días o menos ( $\mathrm{RIQ}=90$ días) (Figura 1). 


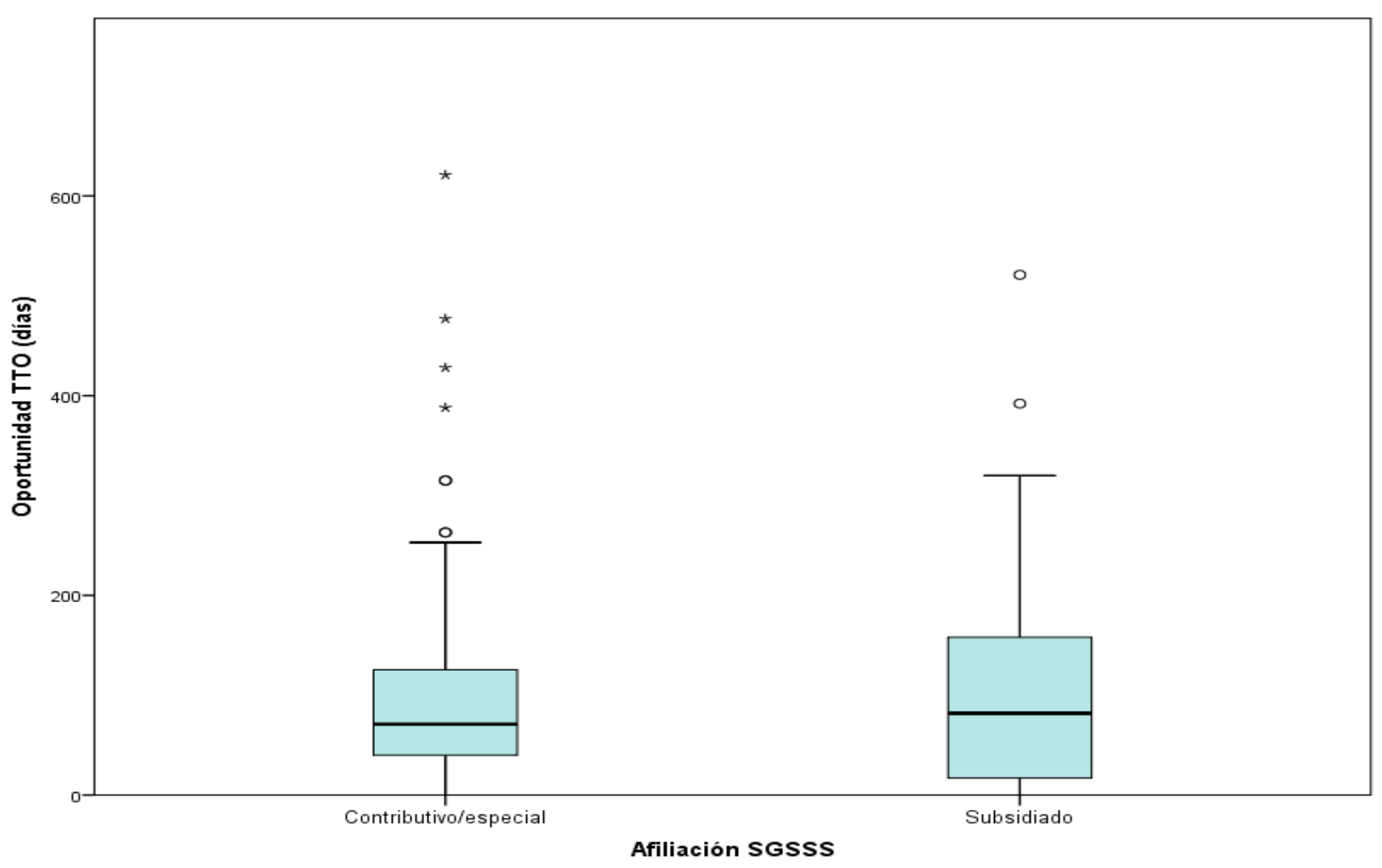

Figura 1. Mediana en días de la oportunidad en el tratamiento, según régimen de afiliación a salud de mujeres con cáncer de mama, Antioquia, 2017.

Se pudo evidenciar que el $50 \%$ de las mujeres pertenecientes al régimen contributivo o especial presentaron una oportunidad en el tratamiento de 71 días o menos, mientras que en las afiliadas del régimen subsidiado fue de 82 días o menos. Sin embargo, no hubo diferencias estadísticamente significativas entre los grupos $(p=0,987)$ (Tabla 1$)$.

Tabla 1. Descripción de los determinantes sociodemográficos de los registros de pacientes con cáncer de mama. Antioquia, 2017.

\begin{tabular}{|c|c|l|l|}
\hline Variables & Categorías & $\mathbf{N}^{\circ}$ & $\mathbf{\%}$ \\
\hline \multirow{2}{*}{ Área de residencia } & Urbana & 338 & 95,2 \\
\cline { 2 - 4 } & Rural & 17 & 4,8 \\
\hline \multirow{2}{*}{ Régimen en salud } & Contributivo/especial & 276 & 78,2 \\
\cline { 2 - 4 } & Subsidiado & 77 & 21,8 \\
\hline \multirow{2}{*}{ Hospitalización } & Si & 10 & 2,8 \\
\cline { 2 - 4 } & No & 345 & 97,2 \\
\hline \multirow{2}{*}{ Condición final } & Viva & 349 & 98,3 \\
\cline { 2 - 4 } & Muerta & $57,0^{*}$ & 1,7 \\
\hline Edad (años) & $5,0^{*}$ & $6,0^{+}$ \\
\hline Oportunidad de diagnóstico (días) & $72,0^{*}$ & $90,0^{+}$ \\
\hline Oportunidad de tratamiento (días) & & \\
\hline
\end{tabular}

*Mediana +Rango intercuartíl (RIQ). 
En el análisis bivariado, se observó que la probabilidad de tener un tratamiento oportuno en las mujeres con cáncer de mama que residen en zona urbana es de 3,08 en comparación con las que viven en zona rural, sin embargo, esta diferencia no es estadísticamente significativa.

En cuanto a la afiliación en salud, se evidenció que el $76,7 \%$ de las mujeres del régimen contributivo recibieron un tratamiento oportuno, mientras que la proporción restante era del régimen subsidiado, siendo esta última condición, un factor que incrementa en un $90 \%$ la probabilidad de tener un tratamiento oportuno al cáncer de mama (RPc: 1,90, IC95\%: 0,81 - 4,46).
Se encontró una asociación significativa ( $p: 0,041)$ entre tener un episodio previo de hospitalización y el tratamiento oportuno a la patología, siendo 9,87 veces más probable en las mujeres con cáncer que fueron hospitalizadas (RPc: 10,87 IC95\%: 1,10 107,39). De otra parte, un diagnóstico tardío ( $\geq 16$ días) disminuye en un $54 \%$ la probabilidad de tener un tratamiento oportuno al cáncer de mama (RPc: 0,46 IC95\%: 0,18 - 1,19). Así mismo, por cada incremento en un año, se disminuye en un $3 \%$ la probabilidad de un tratamiento oportuno (RPc: 0,97 IC95\%: 0,95 - 1,01) (Tabla 2).

Tabla 2. Determinantes demográficos asociados al diagnóstico oportuno en mujeres con cáncer de mama, Antioquia, 2017.

\begin{tabular}{|c|c|c|c|c|c|c|}
\hline \multirow{3}{*}{ Variable } & \multicolumn{4}{|c|}{ Tratamiento oportuno } & \multirow{3}{*}{$\begin{array}{l}\text { RP crudo } \\
\text { [IC 95\%] }\end{array}$} & \multirow{3}{*}{ valor $p$} \\
\hline & \multicolumn{2}{|c|}{ Si } & \multicolumn{2}{|c|}{ No } & & \\
\hline & $\mathbf{N}$ & $\%$ & $\mathbf{N}$ & $\%$ & & \\
\hline \multicolumn{7}{|l|}{ Área de residencia } \\
\hline Urbana & 42 & 97,7 & 136 & 93,2 & $3,08[0,38-24,83]$ & 0,289 \\
\hline Rural & 1 & 2,3 & 10 & 6,8 & 1 & - \\
\hline \multicolumn{7}{|l|}{ Afiliación } \\
\hline Contributivo & 33 & 76,7 & 126 & 86,3 & 1 & - \\
\hline Subsidiado & 10 & 23,3 & 20 & 13,7 & $1,90[0,81-4,46]$ & 0,136 \\
\hline \multicolumn{7}{|l|}{ Hospitalización } \\
\hline $\mathrm{Si}$ & 3 & 7,0 & 1 & 0,7 & $10,87[1,10-107,39]$ & 0,041 \\
\hline No & 40 & 93,0 & 145 & 99,3 & 1 & - \\
\hline \multicolumn{7}{|l|}{ Diagnostico } \\
\hline Oportuno $\leq 15$ días & 35 & 81,4 & 132 & 90,4 & 1 & - \\
\hline No oportuno $\geq 16$ días & 8 & 18,6 & 14 & 9,6 & $0,46[0,18-1,19]$ & 0,111 \\
\hline Edad (años) & 43 & $54^{*}$ & 146 & $58 *$ & $0,97[0,95-1,01]$ & 0,100 \\
\hline
\end{tabular}

Para realizar el análisis multivariado, se incluyó la oportunidad en el tratamiento, como variable dependiente, también se ingresaron las variables área de residencia (rural), afiliación en salud (subsidiado), hospitalización (si), diagnostico (no oportuno $\geq 16$ días) por criterio del investigador. Al realizar el ajuste de las RP mediante el modelo de regresión logística binaria múltiple, se observó que la fuerza de asociación entre la residencia rural y la oportunidad en el tratamiento estaba sobreestimada en el análisis crudo, al compararla con el resultado del análisis ajustado ( $\mathrm{RPa}$ : 2,63
IC95\%: 0,31 - 21,90). Condición similar con el hecho de presentar una hospitalización previa ( $\mathrm{RPa}$ : 8,86 IC95\%: 0,88 - 88,70). Al contrario, la asociación entre la afiliación al régimen subsidiado y la oportunidad al tratamiento de cáncer de mama, estaba subestimada en el análisis crudo, al compararlo con el ajustado (RPa: 1,98 IC95\%: 0,82 - 4,79), donde se observó que la fuerza de asociación aumentó en un $8 \%$. De otra parte, la oportunidad diagnostica tardía, pasó de ser un factor protector en el modelo crudo (RPc: 0,46 IC95\%: 0,18 - 1,19) a ser una condición que 
aumenta la probabilidad de tratamiento oportuno en el modelo final (RPc: 2,01 IC95\%: 0,75 - 5,40). Finalmente, la edad mantuvo su condición en ambos modelos. Sin embargo, ninguna de estas asociaciones fue estadísticamente significativa (Tabla 3).

Tabla 3. Determinantes demográficos que explican la oportunidad en el tratamiento de mujeres con cáncer de mama, Antioquia, 2017.

\begin{tabular}{|c|c|c|c|c|}
\hline Variable & $\begin{array}{c}\text { RP crudo } \\
{[\text { IC 95\%] }}\end{array}$ & valor $\mathbf{p}$ & $\begin{array}{c}\text { RP ajustado } \\
\text { [IC95\%] }\end{array}$ & valor $\mathbf{p}$ \\
\hline Área de residencia (urbana) & $3,08[0,38-24,83]$ & 0,289 & $2,63[0,31-21,90]$ & 0,369 \\
\hline Afiliación en salud (subsidiado) & $1,90[081-4,46]$ & 0,136 & $1,98[0,82-4,79]$ & 0,129 \\
\hline Hospitalización (si) & $10,87[1,10-107,39]$ & 0,041 & $8,86[0,88-88,70]$ & 0,063 \\
\hline $\begin{array}{c}\text { Oportunidad en diagnostico }(\geq 16 \\
\text { días) }\end{array}$ & $0,46[0,18-1,19]$ & 0,111 & $2,01[0,75-5,40]$ & 0,165 \\
\hline Edad (años) & $0,97[0,95-1,01]$ & 0,100 & $0,97[0,95-1,00]$ & 0,115 \\
\hline
\end{tabular}

\section{DISCUSION}

En el presente estudio, la proporción de mujeres con cáncer de mama del departamento de Antioquia que recibieron un tratamiento oportuno fue del $22,8 \%$, cifra muy inferior a reportes internacionales ${ }^{10}$ e incluso locales ${ }^{11}$. Se resalta que, para un tratamiento oportuno, es imprescindible un diagnóstico oportuno, pues mejora el pronóstico y la supervivencia de los casos de cáncer de mama y sigue siendo la piedra angular de la lucha contra este cáncer.

La oportunidad diagnostica en el estudio, tuvo una mediana de 5 días (RIQ: 6 días), cifra que al compararla con la oportunidad diagnostica de países desarrolladas aún es superior ${ }^{12-14}$, pero menor que países como Irán ${ }^{15}$ y Tailandia ${ }^{16}$. Se observó que la oportunidad tardía ( $\geq 16$ días) aumenta la probabilidad de tener un tratamiento oportuno, resultados que son contradictorios a lo reportado por Piñeros et $a l^{17}$ y Sánchez et $a l^{18}$, quién demostró en una categoría que emergió en su estudio cualitativo que las barreras impuestas por el asegurador están relacionadas con la contratación insuficiente de proveedores y prestadores (Contrato con IPS, laboratorios de diagnóstico, proveedores de medicamentos), y con los trámites para acceder a la atención, lo cual, retraso el diagnóstico y por ende el tratamiento.
Una explicación a este resultado contradictorio se debe a los efectos. Esta asociación puede explicarse por la baja potencia estadística debido al tamaño de muestra limitado no permitió comparar ambos grupos. En cuanto a la edad, el estudio de Rodríguez et $a l^{19}$, evidenció que tener más de 50 años es una condición que aumenta la probabilidad de tener un tratamiento oportuno, al igual que el estudio de Gosain et $\left.a\right|^{20}$, lo cual, coincide con los resultados del estudio.

Con respecto a los determinantes demográficos asociados al tratamiento oportuno de las mujeres con cáncer de mama del departamento de Antioquia, se encontró que la residencia urbana aumentaba la oportunidad de tratamiento oportuno. Sin embargo, estos resultados son contrarios a los hallazgos de Charry et $a^{21}$, quienes encontraron que las afiliadas al régimen contributivo tenían menor probabilidad de acceso real a una mamografía para detección temprana que las afiliadas al régimen subsidiado (OR: 0,46; IC95\%: 0,26-0,72) y las pobres sin seguro de salud (OR: 0,36 IC95\%: 0,13-0,65).

De otra parte, un estudio cualitativo realizado en Bogotá por Sánchez et $a 1^{18}$, demostró que las barreras geográficas se mueven en dos escenarios, las que se experimentan en las grandes urbes, y las que se presentan en la ruralidad. Adicionalmente, 
se ven afectadas -de forma diferencial- por el contexto centro versus periferia. Hacen alusión al "fraccionamiento" de los servicios ofrecidos por las aseguradoras en diferentes instituciones en una misma ciudad o en varias ciudades. Esta situación obliga a las mujeres a realizar desplazamientos excesivos dentro de la ciudad o entre diferentes municipios.

Sánchez et $a l^{22}$, encontraron que la pertenencia al régimen contributivo aumentaba la probabilidad de un tratamiento oportuno al cáncer de mama (ORa: 2,96 IC95\%: 1,07 - 10,11), un año después, estos mismos autores, en un estudio crossectional realizado con 136 mujeres con cáncer de mama apoyadas por una organización no gubernamental de Bogotá (11), hallaron resultados similares (ORa: 2,4 IC95\%: 0,63 - 9,05). Pero contrariamente, en este estudio fue la pertenencia al régimen subsidiado el que aumentó esta probabilidad (RPa: 1,98 IC95\%: 0,82-4,79),

Es importante aclarar que el diseño epidemiológico de este estudio, no permite la inferencia causal, por ende, los resultados son aplicables a las mujeres del departamento de Antioquia en un momento puntual de tiempo. Además, la información suministrada, limitó el análisis con otros confusores importantes en el entendimiento de los factores no etiológicos de la oportunidad en el tratamiento y sólo se logró trabajar con determinantes demográficos.

Se resalta que la principal fortaleza del estudio fue la selección completa de los casos, pues, se trabajó con un censo de todos los registros de mujeres con cáncer de mama del departamento en el 2017, situación que permite un mayor control de los sesgos de selección en el estudio. Los resultados de este estudio afirman la necesidad de intervenir desde la promoción de la salud y prevención de las enfermedades, a través de la implementación de acciones educativas, clínicas y sociales enfocadas a controlar las variables demográficas asociadas a la oportunidad en el tratamiento de cáncer de mama. Además, de la participación activa de la ciudadana en la formulación de políticas públicas y fomento de redes de apoyo comunitario.

\section{CONCLUSIÓN}

Los determinantes demográficos que explican la oportunidad en el tratamiento en las mujeres con cáncer de mama en el departamento de Antioquia en el 2017 son, la residencia en zona urbana, la afiliación al régimen subsidiado en salud, la hospitalización previa y el diagnostico tardía ( $\geq 16$ días). Sin embargo, ninguna de estas asociaciones fue estadísticamente significativa.

\section{DECLARACIÓN SOBRE CONFLICTOS DE INTERESES}

Los autores declaran no tener ningún conflicto de interés.

\section{CONTRIBUCIÓN DE LOS AUTORES}

Primer autor: planeación del estudio, diseño metodológico, trabajo de campo, análisis estadístico y redacción del manuscrito.

Segundo autor: planeación del estudio, diseño metodológico, análisis estadístico y redacción del manuscrito

Tercer autor: planeación del estudio, diseño metodológico y redacción del manuscrito

Cuarto autor: planeación del estudio, diseño metodológico y redacción del manuscrito

Quinto autor: planeación del estudio, diseño metodológico, trabajo de campo, análisis estadístico y redacción del manuscrito.

\section{REFERENCIAS BIBLIOGRÁFICAS}

1. Global Cancer Observatory [Internet]. [citado 28 de enero de 2019]. Disponible en: http://gco.iarc.fr/

2. Plan Decenal de Salud Pública [Internet]. [citado 28 de enero de 2019]. Disponible en: https://www.minsalud.gov.co/plandecenal/Pag inas/home2013.aspx

3. Vergara-Dagobeth E, Suárez-Causado A, Gómez-Arias RD, Vergara-Dagobeth $E$, SuárezCausado A, Gómez-Arias RD. Cancer Control Plan in Colombia 2012-2021. A Formal Analysis. Rev Gerenc Políticas Salud. diciembre de 
2017;16(33):6-18. Disponible en: https://www.redalyc.org/html/545/545534160 01/

4. Publicaciones | Instituto Nacional de Cancerologia [Internet]. [citado 28 de enero de 2019]. Disponible en: https://www.cancer.gov.co/publicaciones

5. Richards $M A$, Westcombe $A M$, Love $S B$, Littlejohns P, Ramirez AJ. Influence of delay on survival in patients with breast cancer: a systematic review. Lancet Lond Engl. 3 de abril de 1999;353(9159):1119-26. Disponible en: https://www.ncbi.nlm.nih.gov/pubmed/10209 974

6. Arndt $V$, Stürmer $T$, Stegmaier $C$, Ziegler $H$, Becker A, Brenner H. Provider delay among patients with breast cancer in Germany: a population-based study. J Clin Oncol Off J Am Soc Clin Oncol. 15 de abril de 2003;21(8):1440$6 . \quad$ Disponible en: https://www.ncbi.nlm.nih.gov/pubmed/12697 864

7. Piñeros $M$, Sánchez $R$, Perry $F$, García $O A$, Ocampo R, Cendales R. Demoras en el diagnóstico y tratamiento de mujeres con cáncer de mama en Bogotá, Colombia. Salud Pública México. diciembre de 2011;53:478-85. Disponible en: http://www.scielo.org.mx/scielo.php?script=sci _arttext\&pid=S0036-36342011000600003

8. Abadia CE, Oviedo DG. Bureaucratic Itineraries in Colombia. A theoretical and methodological tool to assess managed-care health care systems. Soc Sci Med 1982. marzo de 2009;68(6):1153-60. Disponible en: https://www.ncbi.nlm.nih.gov/pubmed/19178 990

9. Hansen RP, Vedsted P, Sokolowski I, Søndergaard J, Olesen F. Time intervals from first symptom to treatment of cancer: a cohort study of 2,212 newly diagnosed cancer patients. BMC Health Serv Res. 25 de octubre de 2011;11:284. Disponible en: https://www.ncbi.nlm.nih.gov/pubmed/22027 084
10. Pace LE, Mpunga $T$, Hategekimana $V$, Dusengimana J-MV, Habineza H, Bigirimana JB, et al. Delays in Breast Cancer Presentation and Diagnosis at Two Rural Cancer Referral Centers in Rwanda. The Oncologist. julio de 2015;20(7):780-8. Disponible en: https://www.ncbi.nlm.nih.gov/pubmed/26032 138

11. Sánchez G, Niño CG, Estupiñán C. Factores asociados con el tratamiento oportuno de mujeres con cáncer de mama apoyadas por una organización no gubernamental en Bogotá. Biomédica. 1 de diciembre de 2015;35(4):505$12 . \quad$ Disponible en: http://www.scielo.org.co/scielo.php?pid=S012 0 -

41572015000400008\&script=sci_abstract\&tlng =es

12. Arndt V, Stürmer $T$, Stegmaier $C$, Ziegler $H$, Dhom G, Brenner H. Patient delay and stage of diagnosis among breast cancer patients in Germany -- a population based study. $\mathrm{Br} \mathrm{J}$ Cancer. 8 de abril de 2002;86(7):1034-40. Disponible en: https://www.ncbi.nlm.nih.gov/pubmed/11953 844

13. Burgess CC, Ramirez AJ, Richards MA, Love SB. Who and what influences delayed presentation in breast cancer? $\mathrm{Br} J$ Cancer. abril de 1998;77(8):1343-8. Disponible en: https://www.ncbi.nlm.nih.gov/pmc/articles/P MC2150175/

14. Meechan G, Collins J, Petrie K. Delay in seeking medical care for self-detected breast symptoms in New Zealand women. N Z Med J. 22 de noviembre de 2002;115(1166):U257. Disponible en: https://www.ncbi.nlm.nih.gov/pubmed/12552 275

15. Harirchi I, Ghaemmaghami F, Karbakhsh M, Moghimi R, Mazaherie $H$. Patient delay in women presenting with advanced breast cancer: an Iranian study. Public Health. octubre de 2005;119(10):885-91. Disponible en: https://www.ncbi.nlm.nih.gov/pmc/articles/P MC166160/ 
16. Thongsuksai $\mathrm{P}$, Chongsuvivatwong V, Sriplung $\mathrm{H}$. Delay in breast cancer care: a study in Thai women. Med Care. enero de 2000;38(1):108$14 . \quad$ Disponible en: https://www.ncbi.nlm.nih.gov/pubmed/10630 725

17. Piñeros $M$, Sánchez $R$, Cendales R, Perry $F$, Ocampo R. Patient delay among Colombian women with breast cancer. Salud Pública México. octubre de 2009;51:372-80. Disponible en:

https://www.ncbi.nlm.nih.gov/pubmed/19936 550

18. Sánchez VG, Laza VC, Estupiñán GC, Estupiñán $\mathrm{GL}$. Barriers to healthcare access: narratives of women with breast cancer in Colombia. Rev Fac Nac Salud Pública. diciembre de 2014;32(3):305-13. Disponible en: http://www.scielo.org.co/scielo.php?script=sci _arttext\&pid=S0120-386X2014000300005

19. Rodríguez Ríos $M$, Uscanga Sánchez $S R$, Gutiérrez Vega $P$, Jiménez García GA. Factores asociados a la oportunidad en el diagnóstico de cáncer de mama. Rev Sanid Mil. 31 de octubre de 2017;57(5):291-8. Disponible en: http://www.imbiomed.com.mx/1/1/articulos.p $\mathrm{hp}$ ?method=showDetail\&id_articulo=18000\&id _seccion=88\&id_ejemplar=1879\&id_revista $=16$

20. Gosain R, Pollock Y, Jain D. Age-related Disparity: Breast Cancer in the Elderly. Curr Oncol Rep. 2016;18(11):69. Disponible en: https://www.ncbi.nlm.nih.gov/pubmed/27807 821

21. de Charry LC, Carrasquilla G, Roca S. Equity regarding early breast cancer screening according to health insurance status in Colombia. Rev Salud Pública. octubre de 2008;10(4):571-82. Disponible en: http://www.scielo.org.co/scielo.php?script=sci _arttext\&pid=S0124-00642008000400007

22. Sánchez G, Niño CG, Estupiñán $A C$. Determinantes del tratamiento oportuno en mujeres con cáncer de mama apoyadas por seis organizaciones no gubernamentales en Colombia. Rev Fac Nac Salud Pública. septiembre de 2016;34(3):297-305. Disponible en:

https://aprendeenlinea.udea.edu.co/revistas/i ndex.php/fnsp/article/view/20087/20782733 